\title{
Self-assembly of a new type of periodic surface structure in a copolymer by excimer laser irradiation above the ablation threshold
}

\author{
Carlos Dorronsoro, ${ }^{1, a)}$ Jörn Bonse, ${ }^{2}$ and Jan Siegel ${ }^{1, a)}$ \\ ${ }^{1}$ Instituto de Optica, CSIC, Serrano 121, 28006 Madrid, Spain \\ ${ }^{2}$ BAM Bundesanstalt für Materialforschung und-prüfung, Unter den Eichen 87, 12205 Berlin, Germany
}

(Received 1 August 2013; accepted 30 September 2013; published online 17 October 2013)

\begin{abstract}
We report self-assembly of periodic surface structures in a commercial block copolymer (BCP) (Filofocon A) upon irradiation with a few tens of excimer laser pulses $(20 \mathrm{~ns}, 193 \mathrm{~nm}$ ) at fluences above the ablation threshold. This new type of structures is characterized by much larger periods than those characteristic for Laser-Induced Periodic Surface Structures (LIPSS) and features nanochains instead of ripples. We find a period of $790 \mathrm{~nm}$ at $400 \mathrm{~mJ} / \mathrm{cm}^{2}$, scaling linearly with laser fluence up to a maximum of $1.0 \mu \mathrm{m}$. While an entangled random network of nanochains is produced for normal-incidence and non-polarized light, nanochain alignment can be achieved either by irradiation at an angle or by using linearly polarized light, forming a lamella-like structure. In both cases, the nanochains are aligned parallel to the penetrating polarization orientation and their period does not show a dependence on the angle of incidence, as opposed to the general behavior of standard LIPSS. Also, our results show that the chains are not formed by frozen capillary waves. In contrast, we show analogies of the nanochains produced to lamellar structures fabricated on a smaller scale in other BCP. We discuss the origin of the self-assembly process in terms of a combination of chemical (BCP), optical (surface scattering), and thermal (melting, coarsening, and ablation) effects. (C) 2013 AIP Publishing LLC.
\end{abstract}

[http://dx.doi.org/10.1063/1.4825128]

\section{INTRODUCTION}

Self-assembly of nano- or micrometer-sized objects to form regular, periodic extended structures is a bottom-up technique for nanopatterning, inspired by nature. ${ }^{1,2}$ It is a powerful technology competing with top-down approaches such as lithography, in which a pattern is designed and imprinted by photons, electrons, or ions. When using lasers for patterning, both technologies can be employed. In photolithography (top-down), masks are exposed by a UV laser and projected onto the material to be structured, covered by photoresist, which is then developed and etched. Alternatively, extended periodic structures can be fabricated by means of homogeneous laser irradiation, producing socalled Laser-Induced Periodic Surface Structures (LIPSS) in a mask-free, single-step process that may be considered as bottom-up approach. LIPSS have been discovered already 50 years ago ${ }^{3}$ and have been observed since in semiconductors and metals ${ }^{4}$ and as well as in dielectrics, both inorganic ${ }^{5}$ and polymers. ${ }^{6,7}$ The generally accepted view on the formation mechanism of LIPSS is that the incident laser light interferes with a wave scattered off the sample surface, initially produced by the natural surface roughness and after several pulses due to the gradual formation of LIPSS. The interference causes a modulated intensity distribution, which is imprinted into the material. The model developed by Sipe and coworkers ${ }^{8}$ describes this mechanism and allows predicting the behavior for different materials and different experimental conditions taking into account the complex

\footnotetext{
${ }^{a)}$ Electronic addresses: cdorronsoro@io.cfmac.csic.es and j.siegel@io.cfmac. csic.es
}

refractive index of the material. An essential extension of the Sipe model was developed by Bonse and co-workers, ${ }^{9}$ who took into account the transient complex refractive index of the material during laser irradiation, which strongly differs from the steady-state refractive index due to the formation of a dense electron-hole plasma in the conduction band of the solid. A multitude of experimental results observed by several groups that deviated from the Sipe model could be explained with this modification. Young et al. reported also a different kind of surface structure formed in the high fluence regime. In the third paper of their exhaustive study of LIPSS in semiconductors and metals, they investigate the behavior of LIPSS in different fluence regimes. ${ }^{10}$ The authors classify the structures in different regimes, with regime A being the standard LIPSS type at low fluence, regime $\mathrm{D}$ being the structures formed in the high fluence regime having experienced the homogeneous melting of surface layer, in which capillary waves are formed that subsequently frozen upon solidification, and regimes $\mathrm{B}$ and $\mathrm{C}$ being intermediate types.

We report the formation and self-assembly of periodic surface structures in a copolymer upon irradiation with an excimer laser at $\lambda=193 \mathrm{~nm}$, which cannot be explained by any of the different types of the Sipe-model nor by its extension (taking into account the properties of the excited material). Three key features of these structures differ from LIPSS formed in regime A, which suggests the presence of a different formation mechanism: First, the appearance is strikingly different featuring self-assembled nanochains instead of ripples, and they can also form a random network; second, the period is much larger than the laser wavelength and does 
not depend on the angle of incidence; and third, the nanochains are formed only at fluences well above the ablation threshold, with melting only not being a sufficient condition. We investigate in the present paper the formation of the nanochains structure as a function of laser fluence, angle of incidence, polarization and number of laser pulses and discuss the formation mechanism.

\section{EXPERIMENTAL}

The copolymer sample used was Filofocon A (hydro-2), which is a commercial material (Innovision, Inc., Omaha, $\mathrm{NE}$ ), used to manufacture rigid gas permeable contact lenses. It has also been used successfully as a test material for refractive surgery models upon excimer laser irradiation. ${ }^{11}$ Although the exact composition has not been disclosed, it is a poly-fluoro-silicone-acrylate with a structure composed of five copolymer blocks. ${ }^{12}$ It also contains a cross-linking agent and a wetting agent. The polymer can be classified as a cross-linked block copolymer (BCP), with a molecular weight of 109.96 and a glass transition temperature of $107^{\circ} \mathrm{C}$. For comparison, we have studied the behaviour of a homopolymer; transparent extruded poly(methyl methacrylate) (PMMA, Horniplas, Vitoria-Gasteiz, Spain), whose ablation behavior upon irradiation with excimer laser pulses has been studied by many groups. ${ }^{1,13-15}$ PMMA was chosen since its ablation behavior at the laser wavelength $(193 \mathrm{~nm})$ is relatively similar to that of the copolymer in terms of ablation rate and threshold fluence, as reported in Ref. 11. Both samples are circular slabs (1 $\mathrm{cm}$ diameter, $3 \mathrm{~mm}$ thickness) cut from long bars and polished to optical quality.

For irradiation, we used an ArF excimer laser (LPF200, Lambda Physik, Göttingen, Germany) delivering laser pulses at $\lambda=193 \mathrm{~nm}$ and $20 \mathrm{~ns}$ pulse duration. The laser fluence was continuously adjusted by rotating a coated fused silica window inserted into the beam path, whose transmission depended strongly on the angle of incidence. The laser was operated typically at a repetition rate of $1 \mathrm{~Hz}$ in order to avoid remains of the expanding ablation plume to shield the sample from the next incident pulse. ${ }^{16}$ We have verified though experimentally that the repetition rate does not change the period, alignment, or topography of the surface structures induced within the maximum repetition rate achievable for our laser $(20 \mathrm{~Hz})$.

The beam profile incident on the sample was designed to be top-hat by using an imaging setup. ${ }^{11}$ The latter consisted of a fused silica biconvex lens (focal length $=90 \mathrm{~mm}$ at $193 \mathrm{~nm}$ wavelength) that imaged a $4.5-\mathrm{mm}$ diameter circular aperture, inserted into the beam path $2 \mathrm{~m}$ after the laser output, precisely onto the sample surface. The exact $z$-position of the sample (image plane of the circular aperture plane) was adjusted until sharp edges could be observed on the ablation spot borders. Absolute pulse energy measurements were performed using a calibrated pyroelectric energy detector (Gentec ED 100A). The corresponding laser fluences were calculated by dividing the laser pulse energy by the ablated area and, thus, represent average fluence values.

While most experiments were performed in air with this configuration, providing non-polarized light, some experiments were performed with a modified setup providing linearly polarized light, following the concept used in Refs. 6 and 7. Briefly, the excimer laser beam was polarized by inserting a fused silica prism at Brewster angle in the beam path and selecting the reflected light for irradiation. This configuration eliminates the horizontal polarization components and provides a strongly (although not completely) vertically polarized beam for irradiation. The sample was mounted on a manually controlled $x-y-z$ stage with additional control of the angle of incidence $\theta$.

Sample inspection and characterization were performed with an optical microscope (Nikon Eclipse Ti-E) in reflection, using a $100 \times$ dry objective lens with a numerical aperture of 0.9 and a blue LED illumination source centered at $460 \mathrm{~nm}$ wavelength. The nominal spatial resolution of this system is $310 \mathrm{~nm}$, which is sufficient to identify the periodic structures generated by laser irradiation. In order to obtain high resolution topographic images, we have performed atomic force microscopy measurements on selected regions, using two different systems. The results shown in Figures 1 and 5 have been obtained using an AIST-NT AFM in semicontact mode, whereas for the results displayed in Figure 7(a) Veeco Nanoscope IIIA Multimode in tapping mode has been used.

\section{RESULTS AND DISCUSSION}

We discovered the formation of nanochains accidentally during a comparative study of the ablation behaviour of Filofocon A and PMMA upon excimer laser irradiation, assessing their suitability as test materials for refractive surgery models. ${ }^{11}$ Figures $1(\mathrm{a})-1(\mathrm{~d})$ show AFM images of the progressive formation of nanochains upon irradiation with several laser pulses of unpolarized light and normal incidence at a fluence of $400 \mathrm{~mJ} / \mathrm{cm}^{2}$. As reported in Ref. 11, this fluence is well above the single pulse ablation threshold $\left(F_{\mathrm{a}}=180 \mathrm{~mJ} / \mathrm{cm}^{2}\right)$. The corresponding topography image displayed in Fig. 1(a) was acquired in the center of the ablation spot and shows already the initial stage of a periodic surface structure, featuring agglomerated nanochains with a typical width of $300 \mathrm{~nm}$. The chains appear to be aligned linearly along several directions. We have confirmed experimentally that the initial orientation of the chains after irradiation with one pulse is strongly influenced by pre-existing scratches on the polished sample surface. As in the case of standard LIPSS, also here the surface roughness plays an important role in the ripple formation as it provides the source of the scattering field. ${ }^{4,8}$

After exposure to five pulses, the surface topography has changed notably (cf. Fig. 1(b)). The individual nanochains of $300 \mathrm{~nm}$ width have begun to self-assemble into pairs and form a long, entangled random network of double nanochains. The self-assembly continues upon irradiation with more pulses as can be seen in Figs. 1(c) and 1(d), showing the topography after 10 and 30 pulses, respectively. In the last case (30 pulses), each pair of nanochains has almost completely merged into a single chain of $500 \mathrm{~nm}$ in diameter with lengths up to tens of micrometer.

In order to demonstrate that this surface structure is specific to the copolymer used, we have performed the same 
(a) 1 pulse

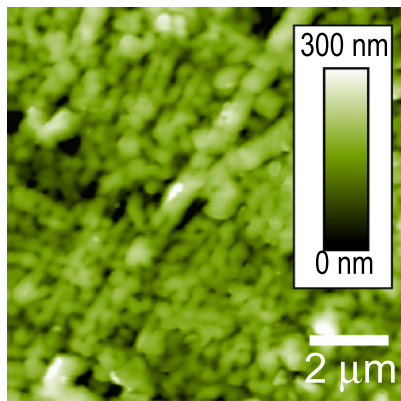

(d) 30 pulses

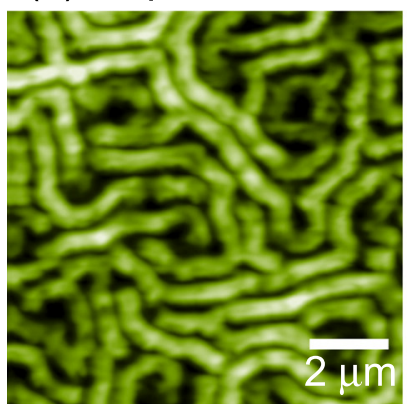

(b) 5 pulses

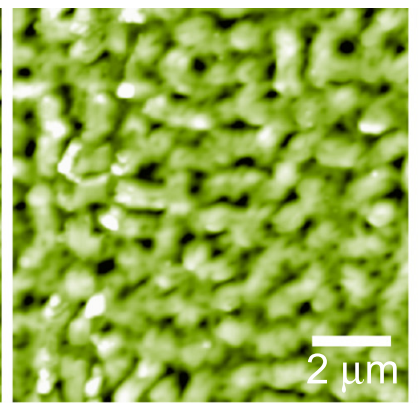

(e) 30 pulses PMMA

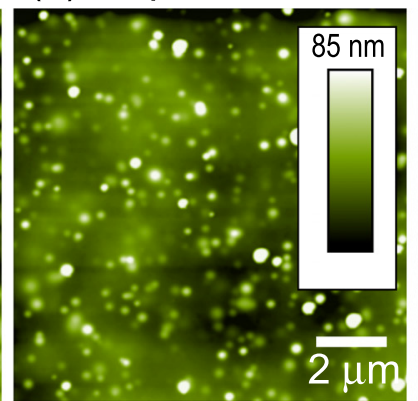

(c) 10 pulses

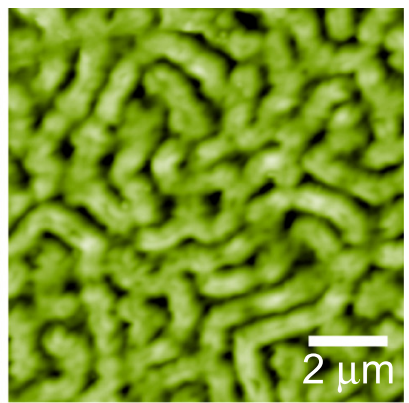

(f) optical

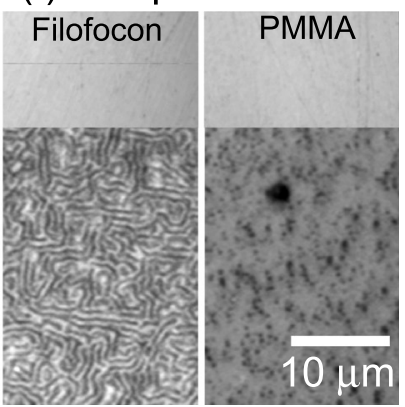

FIG. 1. AFM topography images of Filofocon A (a)-(d) and PMMA (e) polymer surfaces after ablation by nonpolarized $\mathrm{ArF}$ laser pulses at $F=400$ $\mathrm{mJ} / \mathrm{cm}^{2}$ under normal incidence ((a) $N=1$, (b) $N=5$, (c) $N=10$, (d) + (e): $N=30$ ). In (f), large area optical micrographs comparing Filofocon (left panel) and PMMA (right panel) are provided with the upper part of the panels corresponding to unexposed regions, the lower to regions irradiated under the conditions of $((d)+(e))$. experiment in PMMA which has a single pulse ablation threshold of $F_{\mathrm{a}}=280 \mathrm{~mJ} / \mathrm{cm}^{2}$ (Ref. 11). Figure 1(e) reveals the absence of a surface structure, featuring only randomly distributed droplets as sub-micron features. The astonishing length of the chains in Filofocon A and the homogeneous extension of the self-assembled, complex surface structure with a narrow size distribution are demonstrated in Fig. 1(f) providing optical micrographs. We have checked carefully that there is no intrinsic microstructure in the unexposed copolymer. This fact can be seen in the upper part of Fig. 1(f), showing a comparable appearance with low surface roughness of both polymers.

It is worth emphasizing that the chain structure is formed at the crater bottom of the Filofocon A sample. In Ref. 11, we have reported a detailed comparison of the ablation behaviour of both materials. The ablation rate was measured at different fluences and increasing number of laser pulses. At $400 \mathrm{~mJ} / \mathrm{cm}^{2}$, the ablation rate of Filofocon A was found to be constant at $(430 \pm 30) \mathrm{nm} /$ pulse for pulse numbers from 2 up to at least $100 .{ }^{17}$ This value is slightly higher than the thickness of a single chain $(\approx 300 \mathrm{~nm})$, as can be seen in the depth scale in Fig. 1. It is, therefore, surprising that the structure is not erased by the ablation process. We attribute this behavior to the particular ablation mechanism of polymers upon irradiation with UV light, leading to efficient material removal with reduced thermal damage by directly breaking main chain bonds. ${ }^{18}$ However, as pointed out by Dyer, ${ }^{19}$ thermal effects cannot be neglected and will contribute to the decomposition of the polymer. In this context, it is worth noting that the linear absorption coefficient $\alpha$ of Filofocon A $\left(35700 \mathrm{~cm}^{-1}\right)$ is ten times higher than that of PMMA $\left(3800 \mathrm{~cm}^{-1}\right) .{ }^{11}$ As a consequence, the characteristic cooling time $\tau_{c}$ of the hot/molten surface is 100 times shorter, since $\tau_{c}=1 /\left(D \alpha^{2}\right)$, with $D$ being the thermal diffusivity. ${ }^{19}$ For polymers typically $D \approx 1 \times 10^{-3} \mathrm{~cm}^{2} / \mathrm{s}$, which yields $\tau_{c}=0.78 \mu \mathrm{s}$ for Filofocon $\mathrm{A}$ and $\tau_{c}=70 \mu \mathrm{s}$ for PMMA. Moreover, the optical penetration depth $\left(L_{o p t}=1 / \alpha\right)$ is ten times shorter for Filofocon A $\left(L_{\text {opt }}=280 \mathrm{~nm}\right)$. As a consequence, both the time spent at high temperature and the thickness of the heated/molten layer is reduced for Filofocon A. The resulting reduced thermal damage, which is confined to a thin layer, likely leads to the observed "layer-by-layer" ablation of Filofocon A, which is still capable of propagating the chain structure to the underlying layer.

While this certainly contributes to the formation of a defined surface structure, it does not explain its peculiar shape, which is very different from LIPSS formed in regimes A-D of Young and co-workers. ${ }^{8}$ In order to investigate the underlying mechanisms triggering to the formation of the complex network of entangled nanochains in Filofocon A, we have performed a systematic study of the evolution of the structure upon changing the irradiation conditions. Fig. 2 shows optical micrographs of the structures formed upon irradiation with 20 pulses at $400 \mathrm{~mJ} / \mathrm{cm}^{2}$ at different angles of incidence $\theta$. The structure formed at $\theta=0^{\circ}$ (Fig. 2(a)) is a randomly aligned network of nanochains. We have performed a two-dimensional Fast Fourier Transform (FFT) of the image in order to visualize and quantify the periodicity and orientation of the chains. The inset in Fig. 2(a) shows the resulting spectrum of spatial frequencies, featuring a pronounced narrow ring (bright yellow), corresponding to the period of the chains. By changing the angle of incidence to $\theta=20^{\circ}$, the chains become aligned parallel to the plane of incidence (horizontal), as can be seen in Fig. 2(b), now forming a lamella-like structure. This behavior is also observed for larger angles, as shown in Figs. 2(c) and 2(d) for $\theta=40^{\circ}$ and $\theta=60^{\circ}$, respectively. This preferential alignment can be also seen in the corresponding FFT images shown in the insets, featuring isolated peaks as opposed to a ring, which shows that the orientation angle distribution narrows as the 
(a) non-pol. $\theta=0^{\circ}$

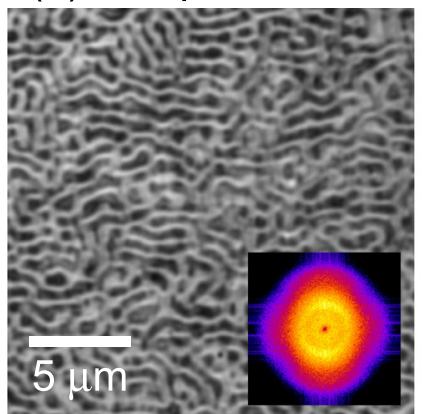

(c) non-pol. $\theta=40^{\circ}$

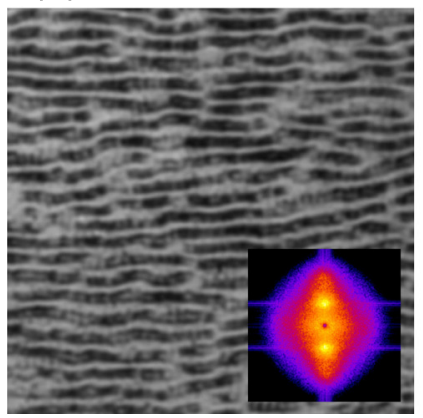

(b) non-pol. $\theta=20^{\circ}$

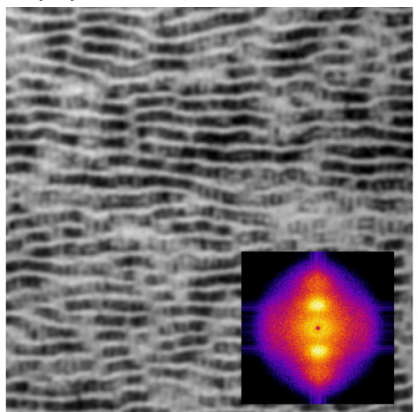

(d) non-pol. $\theta=60^{\circ}$
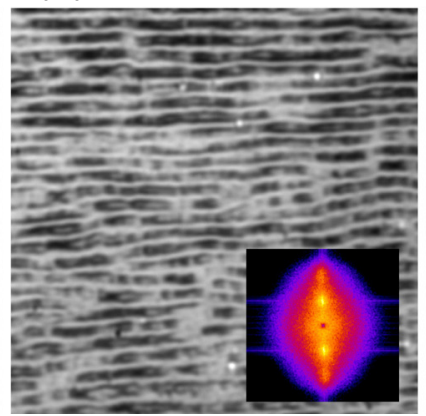

(e)

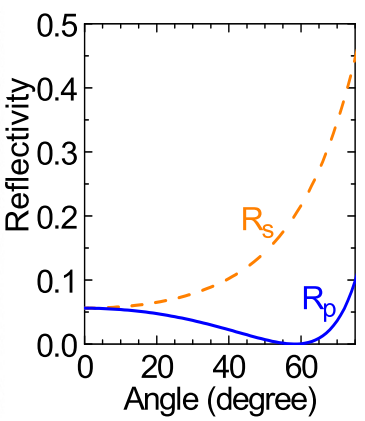

(f) linear pol. $\theta=0^{\circ}$

\section{mi}

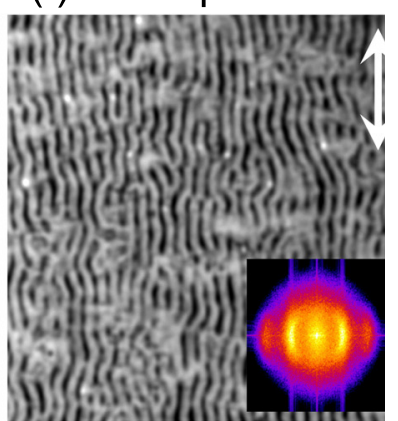

FIG. 2. Optical micrographs of Filofocon A polymer surfaces after ablation by $N=20$ non-polarized $\mathrm{ArF}$ laser pulses at $F=400 \mathrm{~mJ} / \mathrm{cm}^{2}$ under varying angles of incidence ((a) $\theta=0^{\circ}$, (b) $\theta=20^{\circ}$, (c) $\theta=40^{\circ}$, (d) $\theta=60^{\circ}$ ). In (f), the surface was irradiated by linear polarized polarization (direction indicated by arrow). The insets show 2D-FFTs of the corresponding optical micrographs. In (e), the calculated Fresnel surface reflectivity for $s$ - and $p$-polarized radiation at $193 \mathrm{~nm}$ is shown. angle of incidence increases. The weak second-order peak in the FFT images, particularly at $\theta=60^{\circ}$, indicates that that the ratio lamella/space is uneven and deviates from a sinusoidal gray-tone distribution here.

We can extract two conclusions from this behavior. First, the alignment of chains into a lamella-like structure can be controlled optically, which is not only important for applications but also sheds light on the origin of the chain/lamellae formation as will be discussed later. Second, the alignment is directly related to the light polarization. At $\theta=0^{\circ}$, all components of the unpolarized laser light are absorbed in the same way. At larger angles $\theta$, the in-plane ppolarized component of the light is absorbed preferentially, whereas the s-polarized component is mostly reflected. This is confirmed by the calculation of the Fresnel reflectivity of the non-processed polymer surface as a function of the angle of incidence as displayed in Fig. 2(e). At the Brewster angle $\left(58.3^{\circ}\right)$, very close to our experimental situation of $\theta=60^{\circ}$, the p-polarized component is absorbed completely and the spolarized component reflected very efficiently. This explains the narrow angle distribution obtained for $\theta=60^{\circ}$, as can be seen by the narrow horizontal width of the first order of the spatial FFT (cf. Fig. 2(d)). In order to verify this interpretation of the influence of the polarization, we have performed an experiment with linear polarized light (mainly vertical, although not completely; see Sec. II) at $\theta=0^{\circ}$. The result shown in Fig. 2(f) reveals the preferential alignment of the lamellae parallel to the laser beam polarization axis, which confirms the interpretation made for the angle dependence that the lamellar alignment is governed by the penetrating polarization component. It is worth noting that c-type LIPSS found in a few selected materials ${ }^{4}$ also have an orientation of the ripples parallel to the polarization. However, as opposed to our periodic structures, c-type LIPSS are only observed at very high angles $\left(>35^{\circ}\right)$ and never at normal incidence.
Moreover, sometimes LIPSS oriented parallel to the polarization were observed on polymers ${ }^{20,21}$ and low-index inorganic dielectric materials such as silica. ${ }^{22}$ But always the observed LIPSS structures have spatial periods close to the laser wavelength $\lambda$ or $\lambda / n$ ( $n$ : refractive index) for irradiation at normal incidence, which is clearly not the case here.

Most similar to our lamellae, Niino and Yabe ${ }^{23}$ observed periodic surface structures on polyethersulfone (PES) and polyarylsulfone (PAS) upon $308 \mathrm{~nm}$ laser irradiation with multiple $\sim 20$ ns excimer pulses. While they observe comparable periods (between 0.7 and $1.3 \mu \mathrm{m}$ ), the orientation of their structures was perpendicular to laser polarization axis - as opposed to the results presented here.

For the non-polarized laser radiation, we have also studied the progressive lamellae formation at $\theta=60^{\circ}$ as a function of pulse number, as shown in Fig. 3. After one pulse, an initial chain alignment via interference from direct and scattered light can be observed, leading to a lamella-like structure with vertical orientation, perpendicular to the penetrating polarization (Fig. 3(a)). This structure rapidly evolves into a mesh-like structure with vertical and horizontal orientation components after three and five pulses (Figs. 3(b) and 3(c)). After 20 pulses, a well-aligned, lamella-like horizontal structure has been formed, as shown in Fig. 3(d).

In addition to the studies of the influence of the angle of incidence, polarization, and pulse number, we have investigated the influence of the laser fluence. For this study, we have left the other parameters constant; $\theta=60^{\circ}$ and 20 pulses. Figure 4(a) shows that there is a significant increase in the period of the structure, starting from $p=780 \mathrm{~nm}$ at the lowest fluence used $\left(400 \mathrm{~mJ} / \mathrm{cm}^{2}\right)$ and reaching up to $p=1 \mu \mathrm{m}$ at $1.6 \mathrm{~J} / \mathrm{cm}^{2}$. Figures 4(b) and 4(c) display the results obtained from the previous study of the influence of the angle of incidence (for 20 pulses at a fixed fluence of $400 \mathrm{~mJ} / \mathrm{cm}^{2}$ ) and of the pulse number (at $\theta=60^{\circ}$ and a fixed 
(a)

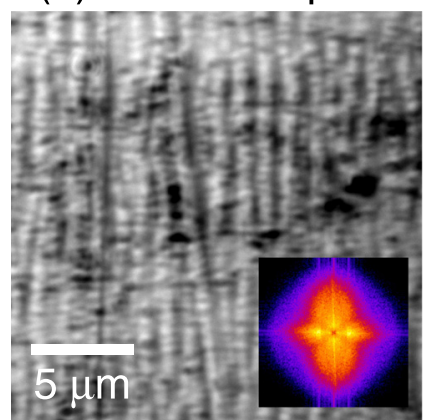

(c)

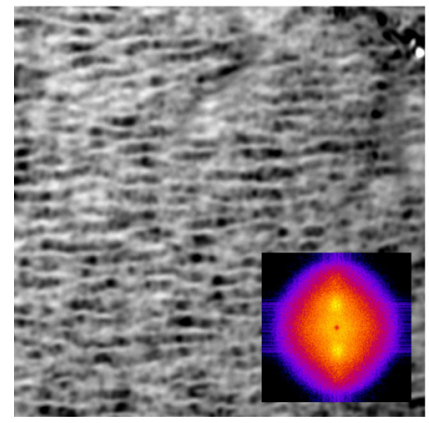

(b)

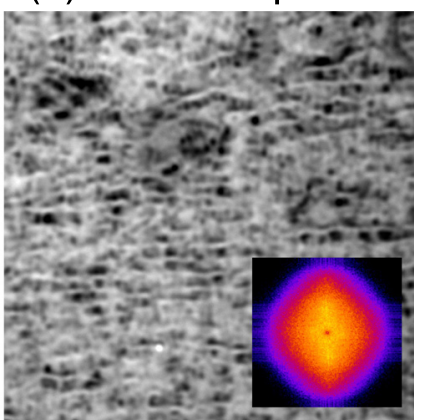

(d)

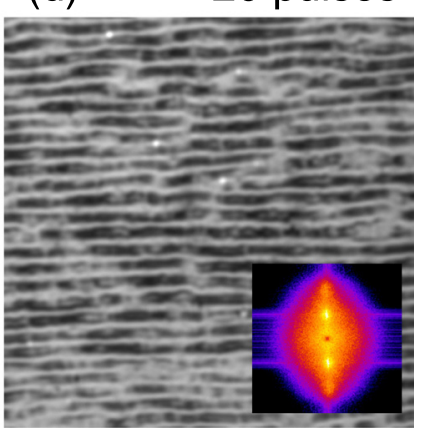

FIG. 3. Optical micrographs of Filofocon A polymer surfaces after ablation by non-polarized $\mathrm{ArF}$ laser pulses at $F=400 \mathrm{~mJ} / \mathrm{cm}^{2}$ and at $\theta=60^{\circ}$ angle of incidence for different pulse numbers $N$ ((a) $N=1$, (b) $N=3$, (c) $N=5$, (d) $N=20$ ). The insets show $2 \mathrm{D}-\mathrm{FFT}$ s of the corresponding optical micrographs.

fluence of $400 \mathrm{~mJ} / \mathrm{cm}^{2}$ ), respectively, summarizing the findings reported in Figs. 2 and 3. Both studies show that there is little influence of these two parameters on the period of the structure, neglecting the data points obtained for very low pulse numbers where the chains/lamellae begin to form. From a practical point of view, we can conclude that these structures can be written reproducibly, homogeneously over a large area with a period that can be selected in the range from $800 \mathrm{~nm}$ up to $1 \mu \mathrm{m}$, at least.

While this study sheds light on the behaviour and controllability of the lamella-like structure upon changes in the irradiation conditions, its peculiar shape (especially for

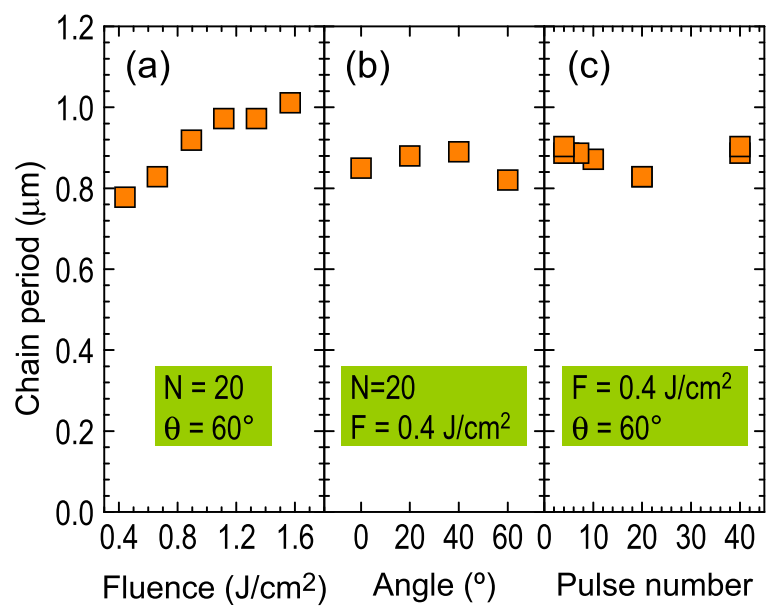

FIG. 4. Chain period versus average laser fluence $F$ (a), angle of incidence $\theta$ (b), and number of laser pulses $N$ (c) after irradiation of Filofocon A polymer surfaces by non-polarized ArF laser pulses. The insets indicate the parameters, which were fixed during the parametric variation.

(a) Filofocon

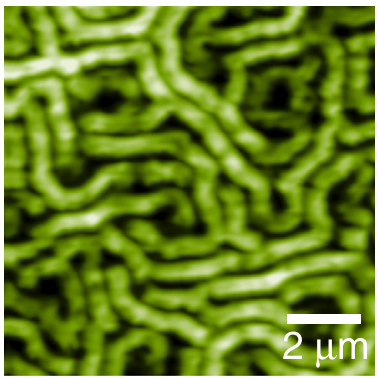

\section{(b) BCP film}

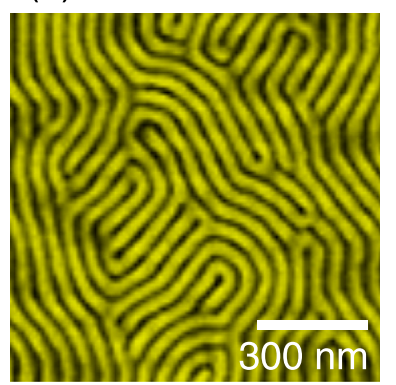

FIG. 5. (a) AFM topography image of a Filofocon A polymer surface after ablation by $N=30$ non-polarized $\operatorname{ArF}$ laser pulses at $F=400 \mathrm{~mJ} / \mathrm{cm}^{2}$ under normal incidence. In order to illustrate the similarity in shape of the lamellar structure to that of self-assembled block-copolymer film (b) shows a SEM image of an annealed PS-b-PMMA film (image taken and adapted from Ref. 25). Note the different magnifications in (a) and (b).

non-polarized light at normal incidence as shown in Fig. 5(a)) is not consistent with any known LIPSS type. A similar shape has been observed, though, in other BCP in solution or in the melt, which can self-assemble upon annealing and form socalled lamellae, driven by the repulsive interaction between the monomers of the different blocks. ${ }^{1,24,25}$ The main difference, however, of the chains/lamellae we observe is their ten times larger diameter $\left(d_{\text {Filo } A}=500 \mathrm{~nm}\right)$, compared to the ones observed in other BCPs, being typically a few tens of $\mathrm{nm}$. Figure 5(b) shows a representative example taken from Ref. 25, in which the $d_{B C P}=50 \mathrm{~nm}$. This value is the natural lamellar spacing of the specific BCP chosen (poly(styreneblock-methyl methacrylate)) and has a defined period $d_{0}=\pi R_{g}$, with $R_{g}$ being the gyration radius of the chains. ${ }^{26} \mathrm{It}$ is a characteristic value for each BCP but never reaches up to values we are observing. However, cross-linking of the chains, as present in our material, may have an impact on the gyration radius of the chains.

Another possible explanation for obtaining such large diameter is that the self-arrangement is not driven by repulsive interaction but by demixing and coarsening found in viscous liquids. Brenier and coworkers studied theoretically the coarsening rates in the demixing process of binary viscous liquids after a temperature quench. ${ }^{27}$ In particular, they investigated how the typical length scale of the domains of the demixed system depends on time, a phenomenon called coarsening. Figure 6(b) displays a result obtained from their model, which has an extraordinary resemblance to our experimental result obtained at $\theta=0^{\circ}$ (Fig. 6(a)). The authors further find that the length scale increases with time, which would correspond in our case to an increase of the time the irradiated polymer remains in the molten phase. Our experimental results, revealing an increase of the period with laser fluence, do support this prediction since it can be safely assumed that the materials remain longer in the molten phase at higher fluences. It is worth pointing out that the process of coarsening is likely to contribute even if there should be no demixing of the constituents of the copolymer. As reviewed in the book on coarsening by Ratke and Voorhes, ${ }^{28}$ also in a single phase system, which is put into a two-phase metastable state, for example, by quenching from a high temperature, a second phase nucleates and grows. Coarsening then 


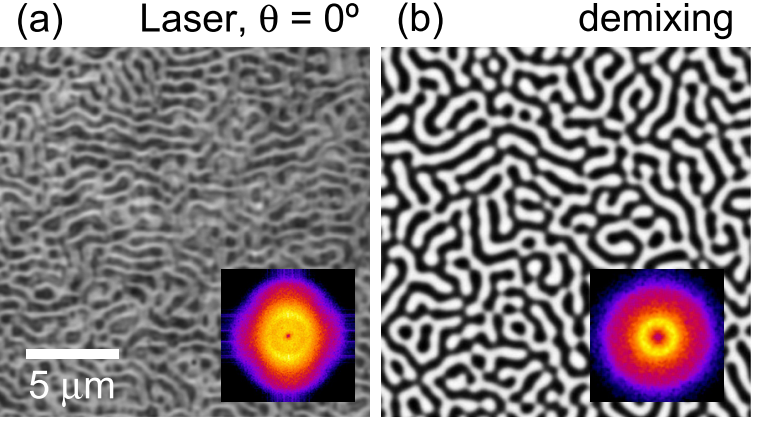

FIG. 6. (a) Optical micrograph of a Filofocon A polymer surface after ablation by $N=20$ non-polarized ArF laser pulses at $F=400 \mathrm{~mJ} / \mathrm{cm}^{2}$ under normal incidence. The insets show 2D-FFTs of the corresponding images. In (b), an example for the calculation of the demixing of a binary viscous liquid after temperature quench is given (taken from Ref. 27).

occurs, where large particles grow at the expense of small particles.

We have also studied the well aligned, lamella-like structures, obtained by irradiation at $\theta=60^{\circ}$, with an AFM. Figure 7 illustrates the extraordinary continuity and alignment these structures have, together with their lamella-like aspect. It emerges clearly from these images that these structures cannot be formed by any known LIPSS process, not even by the one reported by Young et al. for LIPSS in the high fluence regime (regime D), in which capillary waves on a molten layer are frozen. ${ }^{10}$ Figure 7 also displays the phase shift images ${ }^{29}$ of the tapping mode AFM scans, which emphasize the chain-like form of the lamellae but do not provide hints of changes in viscoelasticity, friction, or similar properties. Such changes could show up, for instance, if the copolymer would demix into its constituents and the constituents would have different properties. Yet, the absence of (a) Topography

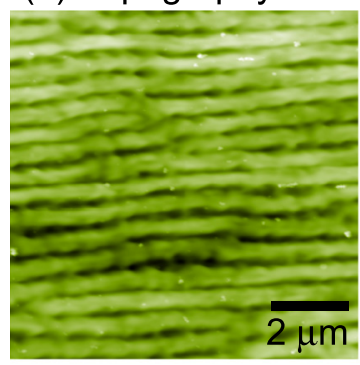

(c) Topography

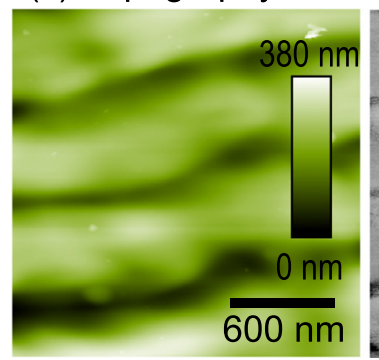

(b) Phase shift

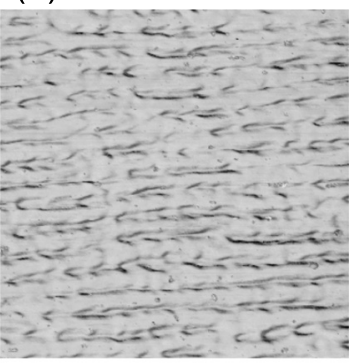

(d) Phase shift

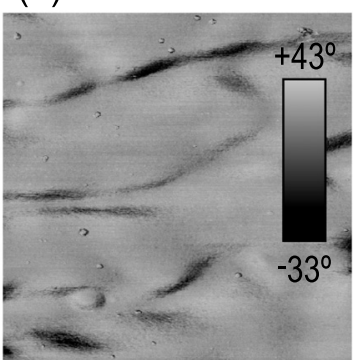

FIG. 7. AFM topography images ((a) and (c)) and corresponding phase shift images ((b) and (d)) obtained in tapping mode at a Filofocon A surface after ablation by $N=20$ non-polarized ArF laser pulses at $F=400 \mathrm{~mJ} / \mathrm{cm}^{2}$ at $\theta=60^{\circ}$ angle of incidence. Images (c) and (d) represent a detailed magnification from the region displayed in (a) and (b). clear features in the phase images does not exclude demixing. And, as mentioned earlier, the coarsening process to which we attribute the formation of chains and lamellae can also occur without demixing.

An open question remains; how exactly does the laser polarization influence the alignment of the lamella. There are studies about optical combing of co-polymers and homopolymers, ${ }^{30}$ which achieve alignment of the lamella by raster scanning a cw beam over the sample. These studies are based on the principle that the chemical reaction rates can be enhanced by increasing the light intensity. In our case, we do have a non-homogeneous illumination field with regions of enhanced intensity due to the interference of the direct laser light with the scattered light, which would, in principle, be consistent with this concept. However, the orientation of the modulation of the light field does not coincide with the alignment of the lamella, which is why there has to be additional factors to influence the alignment of the lamella. One possibility is that the light field of the laser interacts with the electric dipole moment of the molecules in a way to influence molecular ordering and packing. ${ }^{31}$ Further studies are needed to clarify this aspect.

\section{CONCLUSIONS}

We have discovered a new type of surface structure in a copolymer, which progressively self-assembles into nanochains upon laser ablation with a few tens of pulses. The selfassembly can be controlled optically, changing the incident radiation (fluence, number of pulses, incidence angle, polarization). This way, a complex network of entangled long nanochains can be obtained for non-polarized light at normal incidence, or well oriented grating structure with extraordinarily long nanochains for polarized light or angled incidence. The chain period can be tuned from $780 \mathrm{~nm}$ to $1.0 \mu \mathrm{m}$ by increasing the laser fluence and is independent of the angle of incidence or pulse number. The results obtained demonstrate that this type of structure does not belong to any of the different types of LIPSS reported in the literature. The lamella-like structure is very similar to the one found in other block copolymers, although on a ten times longer length scale, which might be due to cross-linking. Additionally, demixing of the copolymer in the molten phase followed by coarsening could favor the formation of nanochains. This approach might pave the way for new, single-step submicron patterning strategies also in other polymers, accessing length scales not accessible by self-assembly of other block copolymers.

\section{ACKNOWLEDGMENTS}

We would like to thank Mikhail Savvateev and Peter Vernhout from AIST-NT for performing the AFM measurements shown in Figures 1 and 5, as well as Daniel Martínez, Aurora Nogales, and Tiberio Ezquerra from the Soft and Polymeric Matter Group at the Instituto de Estructura de la Materia of the CSIC for performing the AFM measurements shown in Figure 7. This work has been partially supported by the Spanish Ministry of Science and Innovation (TEC201122422). 
${ }^{1}$ C. Park, J. Yoon, and E. L. Thomas, Polymer 44, 6725-6760 (2003).

${ }^{2}$ S. Hyde, S. Anderson, K. Larsson, Z. Blum, T. Landh, S. Lidin, and B. W. Ninham, The Language of Shape (Elsevier, New York, 1997).

${ }^{3}$ M. Birnbaum, J. Appl. Phys. 36, 3688 (1965).

${ }^{4}$ J. F. Young, J. S. Preston, H. M. van Driel, and J. E. Sipe, Phys. Rev. B 27, 1155 (1983).

${ }^{5}$ F. Keilmann and Y. H. Bai, Appl. Phys. 29, 9 (1982).

${ }^{6}$ M. Bolle and S. Lazare, J. Appl. Phys. 73, 3516 (1993).

${ }^{7}$ M. Csete and Zs. Bor, Appl. Surf. Sci. 133, 5 (1998).

${ }^{8}$ J. E. Sipe, J. F. Young, J. S. Preston, and H. M. van Driel, Phys. Rev. B 27, 1141 (1983).

${ }^{9}$ J. Bonse, A. Rosenfeld, and J. Krüger, J. Appl. Phys. 106, 104910 (2009).

${ }^{10}$ J. F. Young, J. E. Sipe, and H. M. van Driel, Phys. Rev. B 30, 2001 (1984).

${ }^{11}$ C. Dorronsoro, J. Siegel, L. Remon, and S. Marcos, Opt. Express 16, 20955 (2008).

${ }^{12} \mathrm{M}$. Woodford, personal communication, President of Innovision, Inc., 3125 S 61 st Ave, Omaha, Nebraska 68106, USA (2013).

${ }^{13}$ R. Srinivasan, B. Braren, D. E. Seeger, and R. W. Dreyfus, Macromolecules 19, 916 (1986).

${ }^{14}$ A. Costela, J. M. Figuera, F. Florido, I. Garciamoreno, E. P. Collar, and R. Sastre, Appl. Phys. A 60, 261 (1995).

${ }^{15}$ S. Küper and M. Stuke, Appl. Phys. B 44, 199 (1987).

${ }^{16}$ C. Dorronsoro, S. Schumacher, P. Pérez-Merino, J. Siegel, M. Mrochen, and S. Marcos, Opt. Express 19, 4653 (2011).
${ }^{17}$ Single pulse measurements were excluded because of possible incubation effects and depth resolution limitations.

${ }^{18}$ R. Srinivasan and W. J. Leigh, J. Am. Chem. Soc. 104, 6784 (1982).

${ }^{19}$ P. E. Dyer, Appl. Phys. A 77, 167 (2003).

${ }^{20}$ S. Baudach, J. Bonse, and W. Kautek, Appl. Phys. A: Mater. Sci. Process. 69(Suppl.), S395 (1999).

${ }^{21}$ M. Castillejo, T. A. Ezquerra, M. Martín, M. Oujja, S. Pérez, and E. Rebollar, AIP Conf. Proc. 1464, 372 (2012).

${ }^{22}$ S. Höhm, A. Rosenfeld, J. Krüger, and J. Bonse, J. Appl. Phys. 112, 014901 (2012).

${ }^{23}$ H. Niino and A. Yabe, J. Photochem. Photobiol., A 65, 303 (1992).

${ }^{24}$ P. Alexandridis and B. Lindman, Amphiphilic Block Copolymers: SelfAssembly and Applications, Studies in Surface Science and Catalysis Series (Elsevier, 2000).

${ }^{25}$ S. O. Kim, H. H. Solak, M. P. Stoykovich, N. J. Ferrier, J. J. de Pablo, and P. F. Nealey, Nature 424, 411 (2003).

${ }^{26}$ Y. Tsori and D. Andelman, J. Chem. Phys. 115, 1970 (2001).

${ }^{27}$ Y. Brenier, F. Otto, and C. Seis, SIAM J. Math. Anal. 43, 114 (2011).

${ }^{28} \mathrm{~L}$. Ratke and P. W. Voorhes, Growth and Coarsening. Ripening in Material Processing (Springer-Verlag, Berlin, 2002).

${ }^{29}$ J. Tamayo and R. Garcia, Langmuir 12, 4430 (1996).

${ }^{30}$ R. D. M. Travasso, O. Kuksenok, and A. C. Balazs, Langmuir 22, 2620 (2006).

${ }^{31}$ S. D. Evans, K. E. Goppert-Berarducci, E. Urankar, L. J. Gerenser, and A. Ulman, Langmuir 7, 2700 (1991). 